\title{
Archéopages
}

Archéopages

Archéologie et société

Hors-série 1 | 2008

Construction $^{s}$ de l'archéologie

\section{L’Utile 1761... « Esclaves oubliés »}

\section{Thomas Romon et Max Guérout}

\section{(2) OpenEdition}

Journals

Édition électronique

URL : https://journals.openedition.org/archeopages/851

DOI : 10.4000/archeopages.851

ISSN : 2269-9872

Éditeur

INRAP - Institut national de recherches archéologiques préventives

Édition imprimée

Date de publication : 1 février 2008

Pagination : $59-63$

ISSN : 1622-8545

Référence électronique

Thomas Romon et Max Guérout, «L'Utile 1761... « Esclaves oubliés » », Archéopages [En ligne], Horssérie 1 | 2008, mis en ligne le 01 février 2008, consulté le 23 février 2023. URL : http:// journals.openedition.org/archeopages/851 ; DOI : https://doi.org/10.4000/archeopages.851 
ces produits, ainsi que l'analyse des modalités de fabrication des bracelets, constituent un axe majeur des recherches conduites en Basse-

Normandie [Fig.3].

Le troisième volet de la recherche interrégionale sur le BVSG concerne la chronologie interne du Néolithique ancien. Les corpus mobiliers des sites et les datations sont encore trop limités pour démêler l'écheveau des positions chronologiques de la plupart des gisements. Le cadre chrono-typologique proposé par Claude Constantin fonctionne bien pour la succession «RRBP - BvSG» et le découpage du BvsG; en revanche, l'apparition de certains décors (le V-cordon, en particulier) semble plus ancienne dans l'Ouest que dans le Bassin parisien. Nous ne reviendrons pas ici sur la question des influences méditerranéennes (avec l'Épicardial et le NACA [Néolithique ancien centre Atlantique]) qui ont fait l'objet de nombreux débats (Lanchon 1992; Lanchon, Constantin 1992). Nous souhaiterions cependant réaffirmer le rôle qu'ont pu jouer les régions situées à la périphérie du (ou des) foyer originel du BvsG dans l'évolution interne de celui-ci. La rareté des sites du Néolithique ancien sur la façade atlantique et en Bretagne ne facilite pas la compréhension des données actuellement disponibles. Ainsi les liens entre NACA et Bvsg sont-ils encore trop flous mais ne devraient pas tarder à être explicités.

Affaire à suivre... L'exploration du Néolithique ancien depuis une quinzaine d'année dans l'ouest de la France pose actuellement plus de questions qu'elle n'apporte de réponses à la problématique nationale et aux polémiques sur cette culture. Il serait nécessaire de mettre à plat l'ensemble des données du BvsG et des groupes néolithiques contemporains situés plus au sud.

Une confrontation entre chercheurs de différentes régions serait également constructive. Expliciter la définition du groupe de Bliquy et de VilleneuveSaint-Germain serait un excellent sujet de table ronde. Affaire à suivre, donc...

Augereau A. 1993 : Évolution de l'industrie du silex du $5^{e}$ au $4^{e}$ millénaire avant J.C., dans le Sud-Est du Bassin parisien, Thèse de doctorat, université Paris I.

BAILloud G. 1976: «Le Néolithique en Picardie», Revue archéologique de l'Oise, no7, p.10-28.

Bost yn F. 1994: Caractérisation des productions et de la diffusion des industries lithiques du groupe de Villeneuve-Saint-Germain, Thèse de doctorat, université Paris X.

Bost yn F. (dir) 2003: Néolithique ancien en Haute-Normandie: Le village Villeneuve-Saint-Germain de Poses «Sur la Mare» et les sites de la boucle du Vaudreuil, Société préhistorique française (Travaux 4).

Carpentier V., Marcigny C. 2003: «L'occupation des campagnes entre Évreux et le Vieil-Évreux (Eure): l'exemple des fouilles de la ZAC du Long Buisson», Haute-Normandie Archéologique, $\mathrm{n}^{\circ} 8$, Rouen, Crahn, p.45-51.

Cassen S., Audren C., Hinguant S., Lannuzel G., Marchand G. 1998: «L'habitat Villeneuve-Saint-Germain du Haut-Mée (SaintÉtienne-en-Coglès, Ille-et-Vilaine)», Bulletin de la Société préhistorique française, t. $95, \mathrm{n}^{\circ} 1, \mathrm{p} .41-75$.

Chancerel A., Marcigny C., Ghesquière E. 2006: Le Plateau de Mondeville (Calvados), du Néolithique à l'âge du Bronze, Documents d'archéologie française (DAF), $\mathrm{n}^{\circ} 99$.

Constantin Cl. 1976: «La céramique néolithique et chalcolithique du Bassin parisien et de la vallée de la Meuse, dégraissée à l'aide d'os pilé», in Fouilles protohistoriques dans la vallée de l'Aisne, Paris, vol.4, p.166-172.
Constantin Cl. 1985: Fin du Rubané, céramique du Limbourg et Postrubané en Hainaut et en Bassin parisien, Oxford (BAR International Serie, 273).

Constantin Cl., Demoule J.-P. 1982: «Le groupe de Villeneuve-SaintGermain dans le Bassin Parisien", in Le Néolithique dans l'Est de la France, Actes du Colloque Néolithique de Sens (1980), Cahier de la Société archéologique de Sens, 1, p.65-71.

Constantin Cl., Farruggia J.-P., Guichard Y. 1995: «Deux sites de Villeneuve-Saint-Germain à Bucy-le-Long (Aisne)», Revue archéologique de Picardie, n¹-2, p.3-59.

Clement-Sauleau S., Ghesquière E., Marcigny C. 2002: «Du Ve millénaire au début de l'Antiquité, les occupations de Saint-Vigor-d'Ymonville (76)», Haute-Normandie Archéologique, numéro spécial, Rouen, CRAHN, p.12-17.

Fromont N. 2003: «Anneaux en pierre et culture du VilleneuveSaint-Germain/Blicquy: premiers éléments sur la structuration des productions et la circulation des matières premières entre Massif armoricain et Massif ardennais», in Les Matières premières lithiques en Préhistoire, table ronde internationale (Aurillac 20-22juin 2002), Préhistoire du Sud-Ouest, supplément n ${ }^{\circ}$, p.177-184.

GHESQUiÈre E., MARCignY C. 200o: «Un village rubané en BasseNormandie? L'évaluation du site de la Zac du Lazzaro à Colombelles (Calvados) ", Bulletin de la Société préhistorique française, t.97, $\mathrm{n}^{\circ} 3$, p. 4.05-418.

GirAud P., JuHEL L. 2004: «L'habitat Villeneuve-Saint-Germain de Fontenay-le-Marmion 〈Le Grand Champ > (Calvados)», in Colloque INTERNEO, Paris, $\mathrm{n}^{\circ}$, p. 35-43.

LANCHON Y. 1992: «Le Néolithique danubien dans l'Est du Bassin parisien: problèmes chronologiques et culturels», in Actes du Colloque interrégional sur le Néolithique (Mulhouse, 1984), p.101-117.

LANCHON Y., CONSTANTIN Cl. 1992: «Diachronisme ou synchronisme des groupes de Villeneuve-Saint-Germain et d'Augy-SaintePallaye?», in Actes du Colloque interrégional sur le Néolithique (Mulhouse, 1984), 1992, p.167-175.

Marcigny C., Gaumé E., Ghesquière E., Clement-Sauleau S., Giazzon D., LE GALL J. 2004: «Des centres de production spécialisés à la fin du Néolithique ancien? L'exemple du site de production de parures en schiste de Champfleur «Bois de Barrée (Sarthe)», in P.Bodu et Cl.Constantin (dir.), Approches fonctionnelles en Préhistoire, $\mathrm{XXV}^{\mathrm{e}}$ Congrès préhistorique de France (Nanterre 24-26octobre 200o), Société préhistorique française, p. 265-284.

Patton M., Finlaison M. 2001: Patterns in Prehistoric Landscape, Jersey, éd. La Société Jersiaise.

SEBIRE H. 2005: The Archaeology and Early History of the Channel Islands, Stroud, éd. Tempus.

SIDÉRA I. 1993: Les Assemblages osseux en Bassins parisien et rhénan $d u 6^{e}$ au $4^{e}$ millénaire B.C.: histoire, techno-économie et culture, Thèse de doctorat, université ParisI.

\section{L'Utile 1761... «Esclaves oubliés»}

\author{
Thomas Romon \\ Inrap \\ Max Guérout \\ Groupe de recherche en archéologie navale (Gran)
}

$\mathrm{F}$ in 2006, la mission « L'Utile $1761 . .$. 〈Esclaves oubliés ` » a remis sur le devant de la scène un épisode qui avait déjà défrayé la chronique à la fin du XVIII ${ }^{\mathrm{e}}$ siècle. Le 31 juillet 1761, la flûte L'Utile, propriété de la Compagnie française des Indes orientales, transportant illégalement une centaine d'esclaves, s'échoue sur l'île de Tromelin. Au bout de trois mois, l'équipage abandonne la soixantaine d'esclaves ayant survécu au naufrage et rejoint Madagascar puis l'île de France (l'actuelle île Maurice). Le sauvetage des oubliés de L'Utile aura lieu quinze ans plus tard, le 29 novembre 1776, par l'enseigne de vaisseau le chevalier de Tromelin. Les derniers survivants de ce séjour forcé seront sept femmes et un enfant de huit mois.

Lîle de Tromelin possède un statut juridique bien particulier. Elle est domaine privé de l'État, administrée par le préfet des Terres australes et antarctiques françaises (TAAF). C'est un ancien 


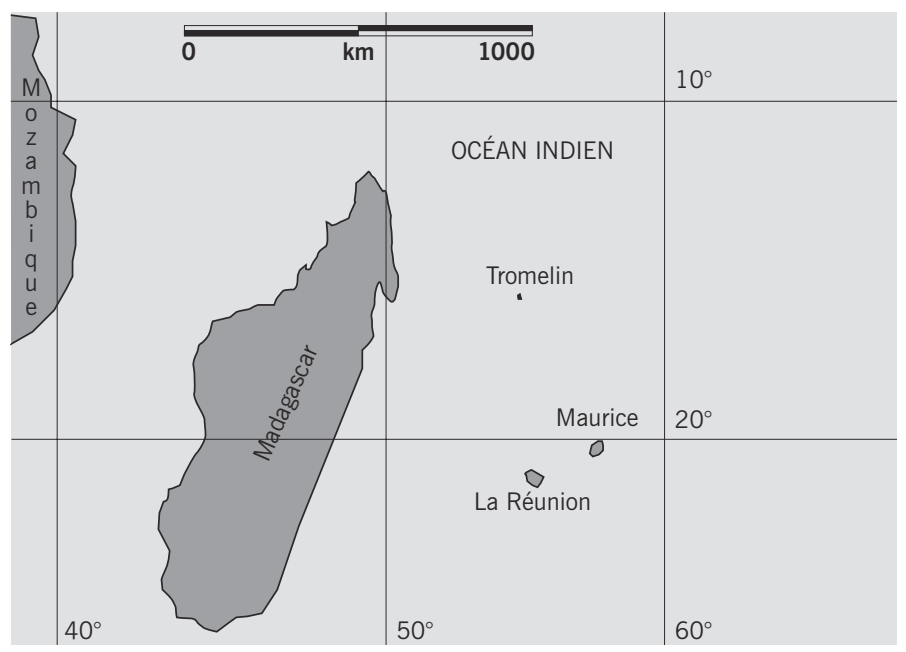

8
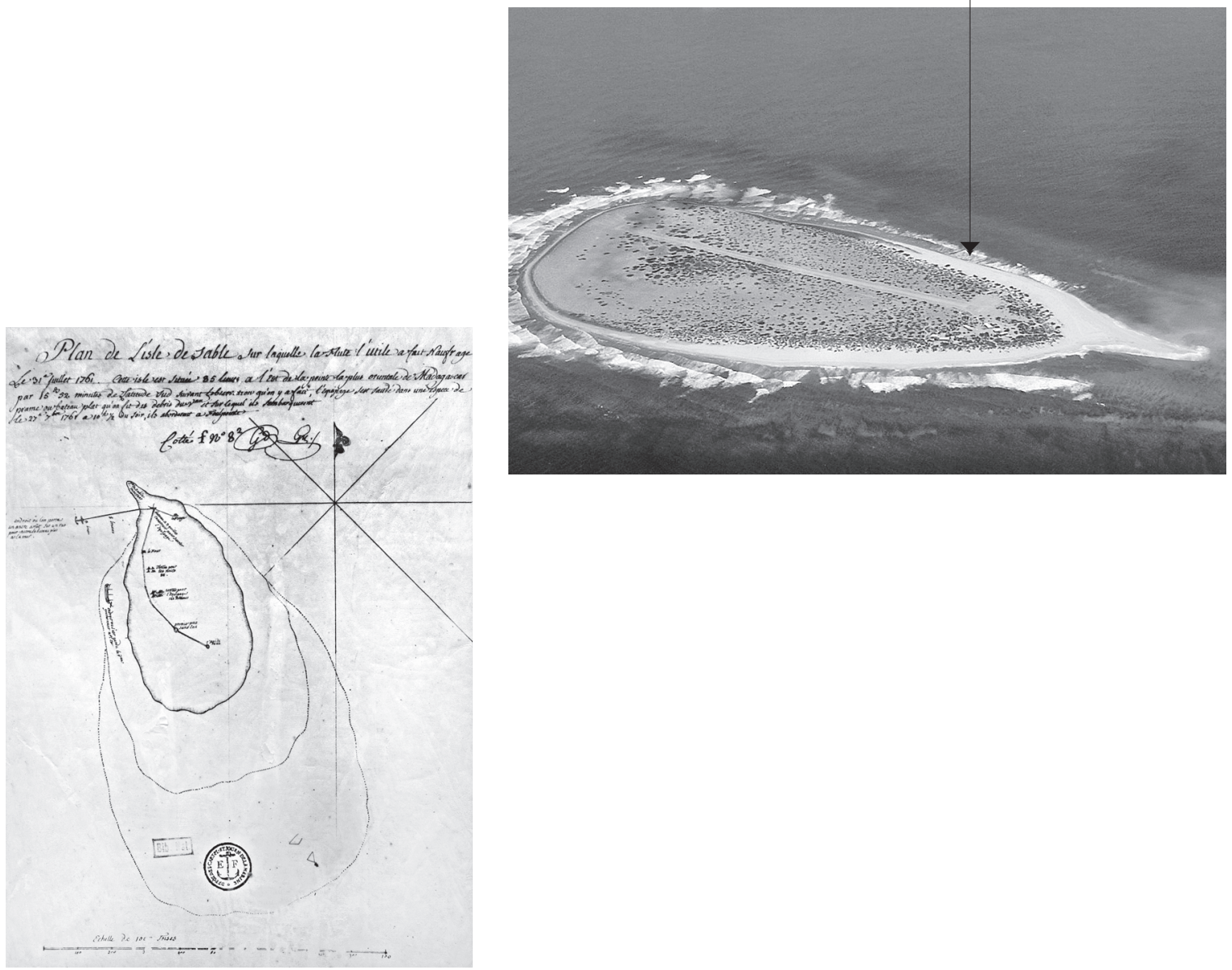

[Fig.1] Localisation de l'île de Tromelin dans l'océan Indien, à $470 \mathrm{~km}$ à l'est de Madagascar et à $560 \mathrm{~km}$ au nord de la Réunion.
[Fig.2] Vue aérienne de l'île de Tromelin depuis le nord-est. On aperçoit les bâtiments de la station météo

sur le point haut de l'île, la piste d'atterrissage. La flèche indique l'emplacement des restes de l'épave.
[Fig.3] Carte de l'île de Tromelin, dessinée le 31 juillet 1761. 
banc de récifs aujourd'hui émergé, développé sur un haut-fond d'origine volcanique. Il est localisé dans l'océan Indien, à 470 kilomètres à l'est de Madagascar et à 56okilomètres au nord de La Réunion [Fig.1]. Il forme une île ovale [Fig.2] d'un peu plus de $1 \mathrm{~km}^{2}$. Le littoral de la partie sud forme un mur de blocs de corail de plus de 1 mètre de hauteur, amoncelés par la houle. La partie nord est constituée de petites dunes de sable corallien. Pratiquement nu, le centre de l'île est légèrement déprimé. Le point le plus haut, situé dans le nordouest de lîle, culmine à 6 mètres. Une bande de récifs coralliens, large d'environ 150 mètres, ceinture lîle. La végétation se limite à quelques arbustes, des veloutiers, auxquels s'ajoutent aujourd'hui quelques cocotiers, aloès et pieds de piment introduits par les agents de la station météo. Il n'y a pas d'eau douce. La faune que l'on rencontre sur lî̂le est surtout constituée d'oiseaux (fous et frégates) et de tortues. Les conditions climatiques y sont assez rudes: vents soutenus engendrés par les alizés, forts courants, houles. Lîle est située sur la trajectoire des cyclones qui traversent l'océan Indien, et une station météo y est établie depuis 1954.

Le projet «Esclaves oubliés», dirigé par Max Guérout, est placé sous le patronage de l'Unesco. Il vise à éclairer, à travers l'étude des conséquences du naufrage de L'Utile, les conditions générales de la traite des esclaves dans l'océan Indien (à Madagascar, en particulier), l'adaptation d'un groupe humain à un environnement nouveau et sa reconstruction sociale. Les recherches historiques [Fig.3], très fructueuses, permettant d'étayer cette mission ont débuté en 2003. Pour la partie terrestre, le Gran a souhaité s'adjoindre les compétences de l'Inrap. Une convention a été signée avec notre institut. Une aide appréciable a été apportée par les agents de Météo France présents sur lîle.

De l'épave, seuls subsistent les éléments les plus lourds (ancres, canons, lest...). Le reste des vestiges a été soit récupéré par les naufragés, soit dispersé par les fortes houles et le courant. L'opération sous-marine a consisté à cartographier des vestiges, ce qui a permis l'identification certaine du navire, corroborée par les archives, et la reconstitution du naufrage. Le choc initial s'est produit sur l'avant à bâbord, vers 4 mètres de profondeur; soit à peu près le tirant d'eau de la flûte (15pieds). Puis, du fait du courant, le navire a pivoté et s'est retrouvé parallèle à la côte, l'avant vers le sud. Pour alléger le bateau, les pièces d'artillerie situées sur le gaillard d'arrière ont été jetées par-dessus bord (concentration de canons). La partie avant du navire s'est détachée et a dérivé pratiquement jusqu'à la plage (deux ancres de bossoir) alors que la partie arrière s'est déplacée de 30 à 40 mètres vers le nord, et s'est orientée perpendiculairement à la côte (concentration de boulets).

L'opération terrestre s'est d'abord tournée vers les secteurs occupés durant la présence des Français. Ils étaient connus au travers des récits et des cartes collectés au retour de l'équipage.
Le four, construit le 15août 1761 pour y cuire le pain et les biscuits nécessaires au retour, a rapidement été localisé par la présence de briques. Il avait été construit à partir des éléments du four du navire échoué. Il ne subsistait de cet édifice que son embase, constituée de blocs de corail assemblés par un mortier de chaux. Un niveau de rejets correspondant très vraisemblablement à une vidange de foyer, situé à 20 centimètres sous le sol actuel, a été échantillonné sur $1 \mathrm{~m}^{2}$. Il a livré des restes de faune (tortue et oiseau) et quelques objets métalliques (petits clous en fer, morceau de plomb). Il est sans équivoque contemporain de l'occupation de 1761.

Les investigations menées sur les autres lieux mentionnés par les archives, en particulier sur le «camp pour les Noirs» et le «camp pour l'équipage», n'ont pas livré de vestiges. Différentes causes peuvent être à l'origine de ce constat: la nature des vestiges, plus ténus que les briques du four; leur localisation plus à l'intérieur de lîle, où la sédimentation est plus faible que sur le cordon dunaire; leur ramassage par les esclaves abandonnés. Par ailleurs, les investigations, uniquement manuelles, ont été assez limitées. Ce dernier point est probablement à l'origine du fait que ni le puits ni les sépultures des naufragés morts dans lîle n'ont pu être trouvés.

Les recherches ont ensuite été orientées vers le secteur occupé par les Malgaches après le départ des Français. Les récits des naufrages postérieurs à celui de L'Utile indiquent la présence d'abris en madrépores situés sur le point haut de lî̂le et attribués à ceux-ci. C'est aussi l'endroit où la station météo a été établie et qui, de ce fait, est très perturbé. Deux sondages, totalisant $20 \mathrm{~m}^{2}$, ont été effectués à proximité de l'ancienne "case malgache», un édifice destiné aux ouvriers malgaches servant les météorologues français, et démoli en 2003. Cet endroit présentait une concentration importante de blocs épars en surface.

Deux niveaux de rejets attribués à l'occupation du XVIII ${ }^{\mathrm{e}}$ siècle, scellés par un niveau de tempête postérieur à l'abandon du site par les naufragés malgaches, ont été mis en évidence à environ 1 mètre de profondeur. Les niveaux de rejets sont constitués de sable, de cendre et de restes consommés, principalement d'oiseaux et de tortues. Les rares restes de poissons confirment la difficulté de pratiquer la pêche depuis le bord de l'île. Pour s'approvisionner en poissons, les marins français avaient construit de petits catamarans qui leur permettaient de pêcher au-delà des déferlantes. Les deux niveaux de rejets sont séparés par un niveau d'abandon à sédimentation marine (sable corallien blanc et grano-classement) caractéristique d'un épisode de tempête. Les vestiges d'une structure en pierres sèches reposent sur ce niveau intermédiaire. Il s'agit des restes d'un mur de 6ocentimètres d'épaisseur, conservé sur à peu près autant de hauteur. L'édifice formé par ce mur n'a pu être identifié. Il s'agit très probablement d'un des abris indiqués par les récits des $\mathrm{XIX}^{\mathrm{e}}$ et $\mathrm{XX}^{\mathrm{e}}$ siècles. 


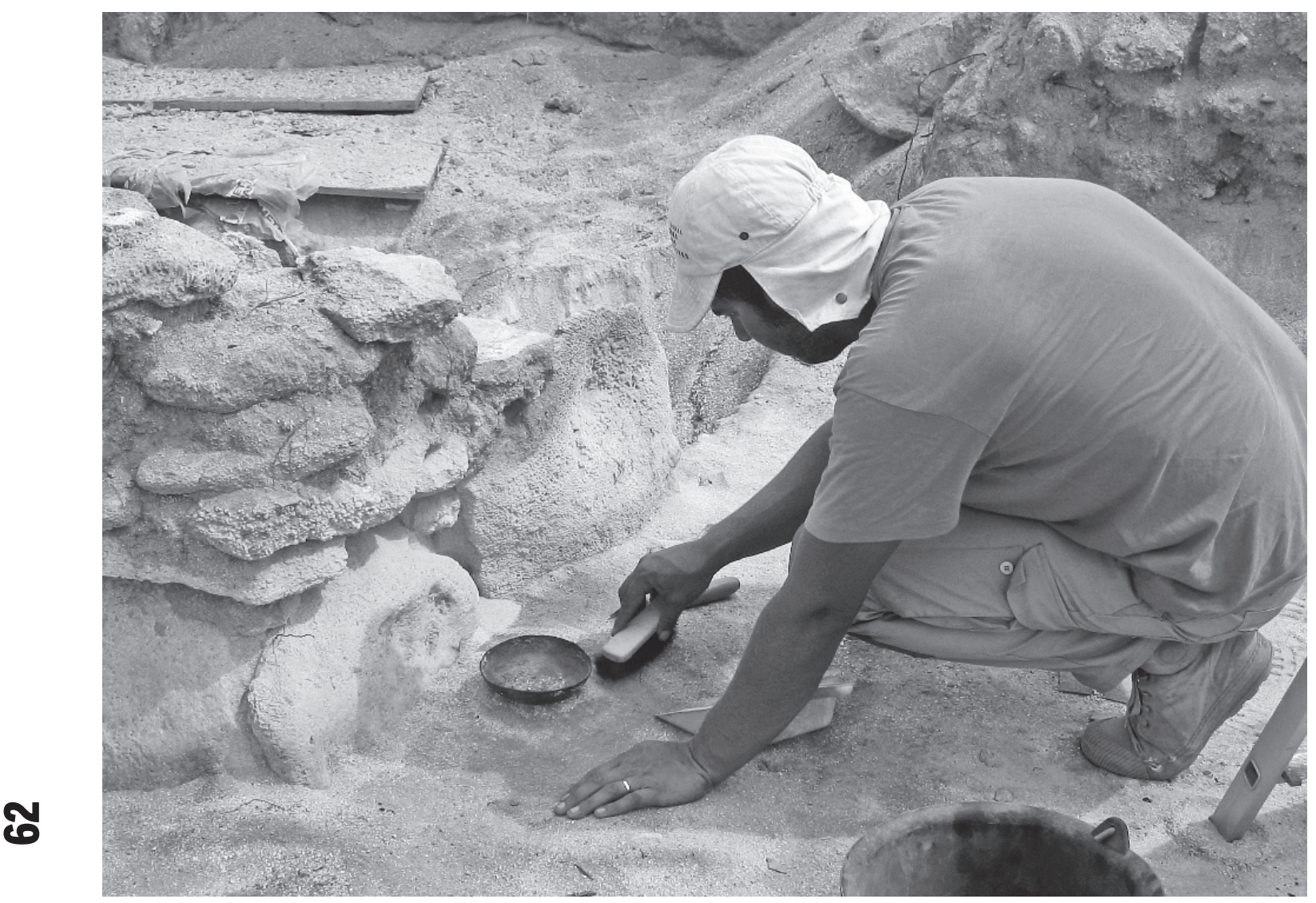

[Fig.4] Fouille des niveaux d'occupation du XVIII ${ }^{\mathrm{e}}$ siècle dans le sondage

du point haut de l'île. On aperçoit

l'un des contenants en cuivre en place au pied des vestiges du mur. 
C'est un épisode climatique assez dynamique, probablement un cyclone, qui, au milieu de leur séjour, a incité les Malgaches à construire des abris en dur. Une partie de celui mis au jour a été réutilisée comme carrière, au moment de la construction de la station météo. Enfin, au sommet du second niveau de rejet et sous le niveau de tempête scellant le tout, reposait en position primaire le mobilier abandonné par les naufragés quand ils ont quitté lîle [Fig.4]. Ce mobilier est constitué de deux contenants en cuivre (quatre autres ont été retrouvés en position secondaire dans un niveau perturbé par l'implantation de la station météo), un galet de lest provenant de L'Utile réutilisé en affûtoir, des clous de navire en fer, des cerclages également en fer et des plaques de cuivre et de plomb. C'est l'un des rares exemples de mobilier utilisé par des esclaves retrouvé dans son contexte archéologique et historique. De plus, les nombreuses réparations portées aux contenants montrent le prix que les naufragés accordaient à ces ustensiles et leur volonté de les utiliser jusqu'à leur extrême usure.

Cette première mission a permis de finaliser une fouille et l'étude de l'épave de L'Utile. Elle a aussi montré la conservation d'indices liés aux quinze années d'occupation des naufragés, confirmant ainsi l'intérêt archéologique de lîle. Cependant, cet aspect archéologique n'a été abordé que très ponctuellement et ne permet pas de répondre de façon satisfaisante à la problématique initiale, en particulier en ce qui concerne le traitement des morts. D'ores et déjà, et dans l'attente d'une seconde mission accompagnée de moyens plus adaptés, des mesures de protection des zones sensibles ont été prises par la préfecture des TAAF.

L'importance de ces vestiges est inestimable. Il s'agit des traces matérielles laissées par un petit groupe humain, déplacé dans un espace vierge. Leur étude permettra d'appréhender les choix effectués par les naufragés dans l'organisation de leur survie. Ces vestiges et leur contexte archéologique devraient nous permettre d'interpréter d'autres sites et de mieux appréhender les dynamiques de peuplement, du milieu insulaire en particulier.

Ces recherches contribuent également

de ce petit point perdu dans l'océan Indien - écueil

\section{Apologie de l'archéologie préventive}

Alain Schnapp

Université Paris I-Sorbonne

$\mathrm{L}$ 'archéologie préventive est née d'un constat élémentaire: les sites archéologiques sont des biens non renouvelables aussi menacés que les espèces naturelles ou les réserves énergétiques. Pendant des centaines de millénaires, l'activité des hommes n'a guère érodé leurs traces. Trop ténues ou trop éparpillées, celles-ci n'étaient pas menacées par les multiples déplacements des chasseurs-collecteurs. La nature, le contexte écologique, les avatars stratigraphiques continuaient à protéger ici ou là ces fragiles niches historiques conservées dans les gorges d'Olduvai, les grottes de Dordogne ou les abords des fleuves, comme à Pincevent.

Avec la sédentarisation, les choses ont lentement changé. Bohumil Soudskỳ avait déjà démontré, il y a presque cinquante ans, que les premiers habitats néolithiques d'Europe centrale correspondaient à des cycles de rotation des cultures et de prélèvements qui alliaient sédentarisation et migrations régulières. Il en avait déduit un modèle de peuplement prédictif qui permettait d'en retrouver les traces lisibles dans le sol. Au Proche-Orient même, les sédentaires ont «inventé» les tells; chaque période en recouvre une autre, en un même lieu. Les premiers théoriciens des ruines, comme Peiresc et Volney, avaient d'ailleurs observé que ces dépôts archéologiques, abandonnés depuis des millénaires, constituaient des ressources de premier plan pour tout historien de l'Antiquité.

Avec les grandes métropoles d'Orient et d'Occident, on a changé d'échelle. Si, comme l'observait Thomas Hobbes, «la papauté n'[était] rien d'autre que le fantôme de l'Empire romain assis couronné sur sa tombe», ${ }^{\mathbf{1}}$ elle habitait un espace, un lieu topographique bien concret que des générations ont transformé en un lieu de mémoire depuis l'Antiquité même. Les Romains étaient conscients de l'érosion rapide des monuments et des traces du passé. De Claude aux empereurs du Bas-Empire, ils s'employèrent à préserver les vestiges de l'Urbs et de sa civilisation. Préservation toute relative... qui faisait place aux constructions nouvelles, aux ambitions édilitaires des empereurs et, même, aux appétits dévorants des spéculateurs (Hoffmann et al. 2005). Cependant, l'existence même d'un débat et la promulgation d'édits impériaux protégeant les monuments sont le signe évident d'un combat entre les exigences du passé et les nécessités du présent. Certaines sociétés ont été plus conservatrices que d'autres, mais aucune n'a fossilisé une fois pour toutes le passé pour en faire une carte si précise et si impérieuse qu'elle eût fini par couvrir le territoire.

Toutes les sociétés ont besoin du passé comme tous les hommes ont besoin de mémoire. Les éradicateurs les plus féroces, comme sur le chemin menant de la métropole aux Indes orientales. Elles rappellent aussi à notre conscience les épisodes peu glorieux du passé esclavagiste et colonialiste de la France. 